\title{
Chancen und Potenziale von Mobility-as-a-Service nach der Corona-Pandemie
}

\author{
Die Pandemie stellt einen Einschnitt in der positiven Entwicklung des öffentlichen Verkehrs \\ dar. Die Furcht der Nutzer:innen vor Infektionen könnte einen nachhaltigen negativen Effekt \\ auf den öffentlichen Verkehr haben. Dieser Beitrag stellt Chancen und Potenziale des \\ Mobility-as-a-Service vor, mit einem Fokus auf die Betriebsform Demand-Responsive Transport. \\ Besondere Chancen könnten in einem Fahrzeugkonzept mit exklusiven Abteilen liegen, die auch \\ bezüglich des Infektionsschutzes Vorteile gegenüber herkömmlichen Fahrzeugen des ÖPNV \\ aufweisen. Auf Basis einer Betriebssimulation werden Anschaffungs- und Besitzkosten ermittelt.
}

\begin{abstract}
Digitalisierung, Flexibilisierung und Automatisierung stellen große Trends in der Mobilitätsbranche dar und ermöglichen gerade im öffentlichen Verkehr die Weiterentwicklung bestehender Strukturen und Konzepte. Im Ergebnis können neue Mobilitätskonzepte neue Nutzungszwecke öffentlicher Verkehrsmittel ermöglichen und damit eine Verkehrsverlagerung bewirken - idealerweise mit positiver Wirkung für Umwelt und Klima. Digitalisierte und flexibilisierte Mobilitätskonzepte wie Mobility-as-a-Service (MaaS) bieten Nutzer:innen neue Möglichkeiten, die eigene Mobilität zu gestalten. Dabei besitzen MaaS-Konzepte Eigenschaften, die sich von klassischen ÖPNV-Linienangeboten unterscheiden. Bei der Diskussion der Auswirkungen der Corona-Pandemie und der damit einhergehenden möglicherweise langfristigen Änderung von Lebensweisen und Präferenzen müssen daher MaaS-Konzepte gesondert betrachtet werden. In der subjektiven Wahrnehmung der Men-

(C) Der/die Autor:in(nen) 2021. Open Access: Dieser Artikel wird unter der Creative Commons Namensnennung 4.0 International Lizenz veröffentlicht (creativecommons.org/licenses/by/4.0/deed.de).

Open Access wird durch die ZBW - Leibniz-Informationszentrum Wirtschaft gefördert.
\end{abstract}

Benedikt Scheier ist wissenschaftlicher Mitarbeiter im Bereich Verkehrsökonomie am Deutschen Zentrum für Luft- und Raumfahrt (DLR).

Benjamin Frieske ist wissenschaftlicher Mitarbeiter am Institut für Fahrzeugkonzepte, Fahrzeugsysteme und Technologiebewertung des DLR.

Kathrin Viergutz ist wissenschaftliche Mitarbeiterin im Bereich Verkehrsökonomie am DLR. schen scheint die Infektionsgefahr in allen Formen des ÖP$\mathrm{NV}$ - unabhängig davon wie stark dieser digitalisiert oder flexibilisiert ist - deutlich größer als z. B. im eigenen Pkw oder beim Radfahren. Dies hat zur Folge, dass eine stärkere Verschiebung der Präferenz hin zu Individualverkehrsmitteln zu beobachten ist. ${ }^{1}$ Mittel- bis langfristig kann dies zu einem Modal Shift weg von Massentransportmitteln und hin zu individuellen Mobilitätslösungen, die eine Distanzierung von anderen Personen erlauben, führen. Hierin könnte eine Chance für MaaS-Angebote liegen. Zu nennen sind flexible, bedarfsgerichtete Angebote des öffentlichen Individualverkehrs, die bestenfalls die positiven Effekte des öffentlichen Verkehrs mit denen des Individualverkehrs kombinieren.

Chancen und Risiken von MaaS im ländlichen Raum

MaaS ist eine bedarfsorientierte Mobilitätsdienstleistung, bei der unterschiedliche Transportoptionen (z.B. ÖPNV, Car-, Bikesharing, Mietwagen, Taxi) in Kombination genutzt werden, um das individuelle Mobilitätsbedürfnis bestmöglich zu befriedigen (Nemtanu et al., 2016). Für Nutzer:innen ergibt sich daraus der Vorteil, dass Mobilitätsdienstleistungen inter- und multimodal über unterschiedliche Verkehrsträger hinweg zentral und anhand von Echtzeitdaten geplant, hinsichtlich unterschiedlicher Kriterien (wie Reisezeit, -kosten, Umweltwirkung) optimiert sowie gesamtheitlich und transparent abgerechnet und bezahlt werden können.

Teil des MaaS-Angebots ist Demand-Responsive Transport (DRT). DRT-Angebote sind Mobilitätskonzepte, die auf dem Prinzip des Ridepooling basieren. Dabei werden räumlich und zeitlich korrespondierende Fahrtwünsche mehrerer Fahrgäste in Echtzeit gebündelt, sodass ein dynamisches Routing entsteht (Ronald, Thompson und Winter, 2015). DRT-Angebo-

$134 \%$ sehen das Auto als Alternative zum ÖPNV und $18 \%$ das Fahrrad (Follmer, 2020). 
Abbildung 1

Chancen und Risiken von MaaS am Beispiel Demand-Responsive Transport im ländlichen Raum

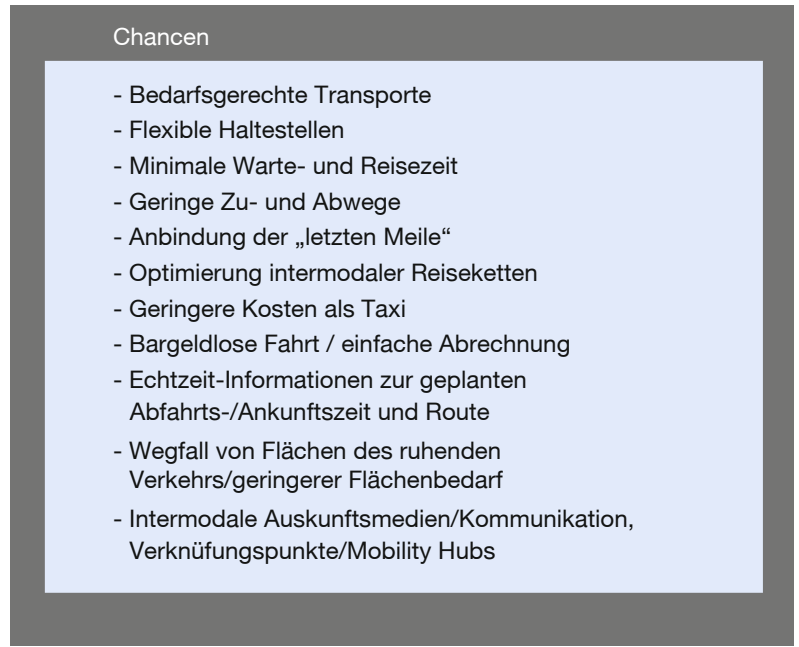

Quelle: eigene Berechnungen.

te vereinen - z.B. durch haltestellenunabhängige Bedienung und den Verzicht auf feste Fahrpläne und Linienwege - die Vorteile individueller und öffentlicher Verkehrssysteme (Laws et al., 2009). Speziell durch einen hohen Grad an zeitlicher Flexibilität und Komfort - wie kurze Wege sowie eine höhere Aufenthaltsqualität - können DRT-Angebote mit den positiven Eigenschaften der privaten Pkw-Nutzung konkurrieren. Zudem bieten DRT-Angebote die Chance, wie gut ausgelastete konventionelle Bussysteme Effizienz-, Kosten- und Umweltvorteile zu erzielen. Dazu zählen z. B. ein im Verhältnis zu den erbrachten Personenkilometern geringeres Verkehrsaufkommen sowie ein geringerer Flächenbedarf ruhender Verkehre im Vergleich zum motorisierten Individualverkehr. Zu beachten ist, dass auch bei DRT-Systemen eine einfache, unkomplizierte und barrierefreie Nutzung ermöglicht sein muss. Betrieblich und wirtschaftlich wünschenswert ist zudem ein hoher Grad an Bündelung, da so die Zahl der eingesetzten Fahrzeuge sowie die insgesamt gefahrene Strecke verringert werden können. Für Nutzer:innen kann die Bündelung von unzureichend deckungsgleichen Fahrtwünschen zu erhöhten Reisezeiten und einer geringeren Servicequalität führen. Daher ist die Definition von Mindeststandards der Servicequalität sinnvoll, durch die notwendige Umwege, verlängerte Reisezeiten sowie Wartezeiten beschränkt werden. In diesem Trade-off aus Nutzer- und Betreiberanforderungen besteht die Chance von DRT-Angeboten. Gleichzeitig stellt dieses Wechselspiel eine der großen Herausforderungen bei der Konzeption von DRT-Bedienkonzepten dar. Es müssen Modelle gefunden werden, in denen ausbalancierte Nutzer- und Betreiberinteressen berücksichtigt werden.

Der ländliche Raum stellt mit seiner starken Zersiedelung und geringen Bevölkerungsdichte eine Herausforderung im Verkehrsbereich dar. Auch und gerade bei DRT-Ange-

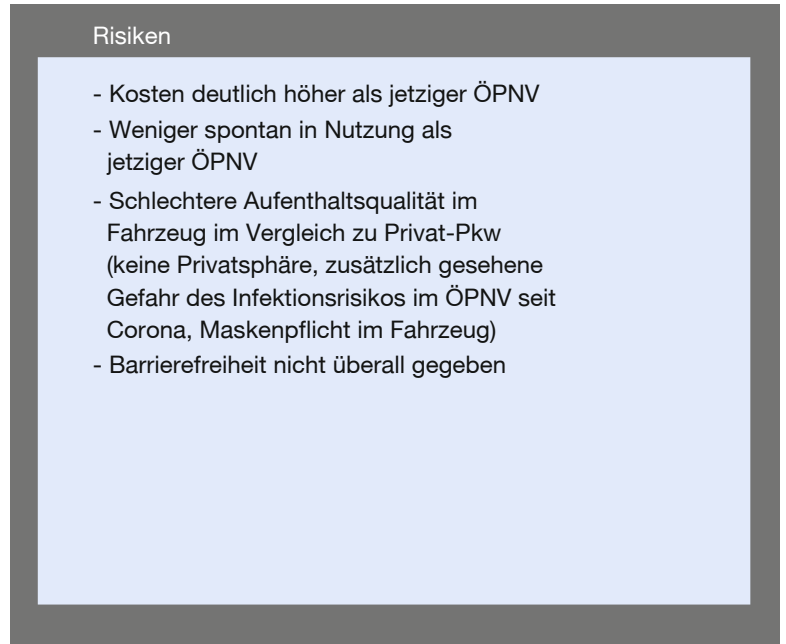

boten, für deren erfolgreichen Betrieb eine entsprechende Bündelungsfähigkeit von Fahrtwünschen gegeben sein muss. Abbildung 1 zeigt ausgewählte Chancen und Risiken von MaaS in ländlichen Räumen.

\section{Einfluss der Pandemie auf Mobilitätsgewohnheiten}

Die Corona-Krise stellt einen deutlichen Einschnitt in der Verkehrsentwicklung dar. Zu Beginn der Pandemie hat sich die Alltagsmobilität vieler Menschen abrupt verändert. Kurzfristig wurden deutlich weniger Wege zurückgelegt. Doch auch während der über die Zeit der Pandemie schwankenden Intensitäten der Ansteckungsgefahr sowie sich ändernden Einschränkungen zeigen sich Veränderungen im Mobilitätsverhalten. Auch wenn abzusehen ist, dass sich nach der Pandemie viele Gewohnheiten und Lebensbereiche wieder normalisieren, so ist doch mit veränderten Mobilitätsgewohnheiten zu rechnen. Insbesondere bei den öffentlich zugänglichen, kollektiven Verkehrsmitteln ist eine Veränderung zu erwarten. Dabei sind im Wesentlichen zwei Auslöser zu unterscheiden: Zum einen könnten Menschen die Gewohnheit entwickelt haben, auch langfristig dem Kontakt zu anderen Menschen kritisch gegenüberzustehen und daher den Aufenthalt an Orten mit hohem Personenaufkommen bewusst einzuschränken. Zum anderen kann sich der Verkehrsbedarf durch veränderte Lebensumstände verringern, z. B. durch den Rückgang von Pendlerverkehren und Dienstreisen (Homeoffice sowie digitalisierte Veranstaltungen).

Während der ersten Phase der Pandemie im Frühjahr 2020 hat sich ein deutlicher Trend weg von kollektiven Verkehrsmitteln hin zu Individualverkehrsmitteln gezeigt, der auch derzeit noch anhält. So haben viele Menschen das Fahrradfahren als Mobilitätsmöglichkeit für sich entdeckt oder 
in die Anschaffung eines eigenen Pkw investiert. Auch viele Fahrgäste des öffentlichen Verkehrs, die bisher auf den ÖPNV angewiesen waren, finden nun andere Lösungen zur Deckung ihres Mobilitätsbedarfs und kehren möglicherweise nach der Pandemie nicht wieder zu bisherigen Nutzungsmustern zurück. So könnte sich langfristig eine veränderte Verkehrsmittelwahl und damit eine Verschiebung des Modal Split einstellen.

Neue Rahmenbedingungen im Alltag und in der Arbeitswelt machen neue Ansätze der eigenen Verkehrsmittelwahl notwendig. Langfristig kann aus der plötzlichen Verlagerung von Geschäftstätigkeiten ins Homeoffice die Möglichkeit erwachsen, auch nach der Pandemie einen Teil der Arbeit zu Hause zu verrichten. Dabei sind auch Modelle wahrscheinlich, bei denen Mitarbeiter:innen langfristig ihren Wohnort deutlich stärker als zuvor vom Sitz der Arbeitsstelle trennen. Z.B. zieht eine Person an einen weiter entfernten Wohnort um, um im Homeoffice zu arbeiten und nur noch gelegentlich an den Firmenstandort zu pendeln. Aus dem Wandel weg vom täglichen Pendeln hin zur gelegentlichen Bewältigung von Langstrecken (für Präsenztermine) kann sich ein veränderter Verkehrsbedarf ergeben. Im Wesentlichen zeichnen sich dabei drei Trends ab:

- Verstärkte Nahmobilität im eigenen Stadtteil (z.B. Besorgungen im Supermarkt für die Verpflegung im Homeoffice)

- Reduktion mittellanger Strecken (z. B. Arbeitswege)

- Neuer Langstrecken-Gelegenheitsverkehr (z. B. gelegentlich zur Arbeitsstätte)

Diese Entwicklung kann in einer Verdichtung ländlicher Räume sowie in verändertem Mobilitätsbedarf resultieren.

\section{Einfluss der Pandemie auf die Entwicklung von DRT}

Kurzfristig haben die neuen Lebensumstände zu deutlichen Verschiebungen in der Verkehrsmittelwahl geführt, die sich langfristig etablieren könnten. Zudem zeigt sich das Bedürfnis der Menschen nach Verkehrsmitteln, durch die ein geringer Kontakt zu Mitmenschen gewährleistet ist. Hierin kann eine Chance von DRT-Angeboten liegen, sofern diese zudem auch flexibel und zuverlässig sind.

Ein Ansatz bei der Gestaltung von entsprechenden Mobilitätskonzepten liegt in der Entwicklung unterschiedlicher Fahrzeugeinheiten. Durch eine fahrzeugbauliche Gestaltung, die eine räumliche Abtrennung von anderen Fahrgastgruppen erlaubt, kann Nutzer:innen das Gefühl einer angemessenen Privatsphäre und gesundheitlicher Sicherheit zurückgegeben werden. Ein DRT-Abteilbus erlaubt die exklusive Nutzung eines Abteils durch einzelne Fahrgäste bzw. Fahrgastgruppen mit einer verringerten Ansteckungsgefahr.

\section{Chancen von DRT im ländlichen Raum}

DRT steht für ein Verkehrssystem, das Fahrten für den Transport von Personen nach Bedarf der Reisenden zur Verfügung stellt und nicht an einen Fahrplan oder vorab festgelegte Fahrtrouten gebunden ist. Von einem Dispositionsalgorithmus werden zeitlich und räumlich ähnliche Fahrtanfragen gebündelt, sodass die Auslastung der Fahrzeuge erhöht werden kann - mit positivem Effekt auf Emissions- und Ressourcenverbrauch - und gleichzeitig die Chance besteht, den Fahrpreis im Vergleich zur Nutzung anderer Mobilitätsdienstleistungen (z. B. Taxi) zu verringern. Jedoch besteht das Risiko, dass die Fahrtzeit durch die Bündelung von Fahrten mit unterschiedlichen Start- und Zielpunkten steigen kann. Gerade in ländlichen Räumen mit schwacher Verkehrsnachfrage haben fahrplanbasierte Linienverkehre den Nachteil, dass das Angebot aufgrund wirtschaftlicher Zwänge stark eingeschränkt ist.

Im Rahmen einer Simulationsstudie wurden die Reiseund die Wartezeiten auf eine Fahrt sowie die benötigte Fahrzeuglaufleistung ermittelt. Für die Simulation mit der Verkehrsflusssimulation (Simulation of Urban Mobility, SUMO) (Alvarez Lopez et al., 2019) wird ein ländlicher Untersuchungsraum mit einer Fläche von ca. 600 km² konstruiert. Dieser besteht aus einer großen Zahl ungefähr gleich verteilter Siedlungen und einer Kleinstadt in der geografischen Mitte. An den Simulationsraum angrenzend befinden sich zwei weitere Städte sowie eine Großstadt, die mit dem ländlichen Raum über je eine zentrale Haltestelle verbunden sind. Im ländlichen Raum zwischen diesen Städten bietet das DRT jede Fahrtrelation an und entspricht damit einer Angebotsverbesserung gegenüber dem herkömmlichen fahrplan- und linienbasierten ÖV.

Soweit nicht ein Taxi gebucht wird, müssen die Reisenden den Raum eines Fahrzeugs miteinander teilen. Zumeist wird dies aufgrund geringerer Privatsphäre als negativ empfunden; seit der Pandemie ist zudem ein (zumindest subjektiv empfundenes) erhöhtes Infektionsrisiko vorhanden. Um diesen DRT-Nachteil auf ein Minimum zu begrenzen bzw. ähnlichen Komfort wie im eigenen Pkw zu bieten, werden zwei unterschiedliche DRT-Fahrzeugtypen analysiert: ein herkömmlicher Kleinbus und ein neuartiger Abteilbus. Inwiefern sich die Kosten der Anschaffung und des Betriebs von Abteilbussen zu Kleinbussen unterscheiden, wird durch eine Verkehrssimulation des DRT-Betriebs, ein ökonomisches Modell zur Ermittlung der Anschaffungskosten und eine angeschlossene betriebswirtschaftliche Analyse betrachtet.

\section{Verkehrssimulation eines DRT-Betriebstags}

Mit der Verkehrsflusssimulation SUMO wird der DRTBetrieb eines Tages von 05:00 bis 23:00 Uhr simuliert. Es 
wird angenommen, dass mit ca. 4.300 Fahrtanfragen eine typische Nachfrage an öffentlichen Verkehren im ländlichen Raum an einem Werktag vorliegt. Die werktägliche Nachfrage wird anhand einer für den ländlichen Raum typischen Tagesganglinie auf einzelne Stunden heruntergerechnet. Innerhalb einer Stunde wird eine Gleichverteilung angenommen. Die räumliche Verteilung der Nachfrage wird anhand von Verkehrsmengen auf Gemeindeebene eines bestehenden Nahverkehrsplans angenommen und anhand der Bevölkerungszahlen auf die einzelnen Dörfer verteilt. Die fahrtanfragenden Personen gehen von ihrem Startort zu einer ÖV-Haltestelle, setzen die Fahrtanfrage ab, warten auf das DRT-Fahrzeug, werden zur Haltestelle nahe dem Zielort gefahren und gehen von der Haltestelle zum Ziel. Der Dispositionsalgorithmus versucht bei der Zuweisung einer Fahrtanfrage zu einem freien Fahrzeug eine weitere Fahrtanfrage mit zuzuweisen, soweit die daraus resultierende neue Fahrtzeit nicht das Doppelte des Ursprünglichen überschreitet. Weitere Personen, deren Fahrtanfrage an Haltestellen entlang einer durchgeführten Route liegen und die im selben Zeitraum bestehen, werden den bereits bestehenden Fahrtanfragen zugeordnet. Weitere Verkehre wie der ÖPNV, der Individualverkehr oder der Güterverkehr wurden in dieser ersten Simulation nicht berücksichtigt. Auch wurden Zeiten für den Fahrgastwechsel und das Laden der Batterie in der Simulation nicht berücksichtigt. Hier wird angenommen, dass die Betriebspause von 23 bis 5 Uhr ausreicht, um den simulierten Betrieb gewährleisten zu können. Diese Punkte sollen in den zukünftigen Simulationen verfeinert werden, wie auch die weitere Verbesserung des Dispositionsalgorithmus, um die Mitnahme weiterer Personen bei möglichst wenig zeitlichen bzw. räumlichen Umwegen zu ermöglichen.

Tabelle 1 zeigt die Ergebnisse von drei Simulationsläufen, in denen nur die Zahl der zur Verfügung stehenden DRTFahrzeuge variiert wurde. Bei sinkender Fahrzeugzahl steigt die Bündelung von Fahrten stark, und somit erhöhen sich auch die Wartezeiten. Da der Dispositionsalgorithmus lediglich geringe zeitliche Umwege in Kauf nimmt, steigt die Fahrtdauer bzw. Strecke nur sehr wenig und auch der Besetzungsgrad ist besonders bei hoher Fahrzeugzahl gering. Bei sinkender Fahrzeugzahl sinken die Standzeiten der Fahrzeuge erheblich. Jedoch steigt die Leerfahrtleistung im Verhältnis stärker als die Fahrtleistung mit Personen durch vermehrte Fahrtenbündelung sinkt.

\section{Ermittlung der Anschaffungskosten}

Für die Ermittlung der Fahrzeuganschaffungskosten und der betriebswirtschaftlichen Analyse werden zwei Fahrzeugtypen betrachtet. Ein Kleinbus mit sechs Sitzplätzen, der den Vorzug bietet, dass er klein und leicht ist. Nachteilig ist, dass die Sitzplätze der Reisenden, die sich die Fahrt teilen, auf einer relativ geringen Fläche verteilt sind. Zudem wird ein Ab-
Tabelle 1

Simulationen eines Demand-Responsive TransportBetriebstags mit unterschiedlicher Fahrzeugzahl

\begin{tabular}{lrrc} 
& \multicolumn{3}{c}{ Zahl DRT-Fahrzeuge } \\
\cline { 2 - 4 } Indikatoren (ein Betriebstag) & 175 & 100 & 80 \\
\hline Beförderte Personen & 4200 & 4200 & 4200 \\
\hline Personenkilometer & 61.700 & 61.700 & 63.400 \\
\hline Fahrtenbündelung (\%) & 43 & 60 & 97 \\
\hline Max. Zahl Reisende einer Fahrt & 4 & 5 & 10 \\
\hline Mittlere Zahl Reisende je Fahrzeug & 1,28 & 1,47 & 2,25 \\
\hline Mittlere Wartezeit auf Fahrt (min.) & 5 & 11 & 38 \\
\hline Standardabweichung (mittlere Wartezeit) & 6,4 & 10,81 & 25,63 \\
\hline Mittlere Fahrtdauer (min.) & 14,5 & 14,8 & 15,1 \\
\hline Mittlere Fahrtstrecke (km) & 14,7 & 14,7 & 15,1 \\
\hline Mittlerer zeitlicher Umwegfaktor wegen & 1,032 & 1,033 & 1,042 \\
\hline Fahrtenbündelung & 66.000 & 70.000 & 71.000 \\
\hline Fahrzeug-Strecke (gesamt) (km) & 56.000 & 52.000 & 46.000 \\
\hline Fahrzeug-Strecke (besetzt) (km) & 8.200 & 17.500 & 25.000 \\
\hline Fahrzeug-Strecke (leer) (km) & 654 & 411 & 175 \\
\hline Mittlere Standzeit (min.) & &
\end{tabular}

${ }^{1}$ Fahrten mit mindestens zeitweise zwei Reisenden.

Quelle: eigene Berechnungen.

teilbus mit drei räumlich voneinander getrennten Abteilen mit bis zu sechs Sitzplätzen eingesetzt. Drei Sitze sind klappbar, sodass bei maximal drei sitzenden Reisenden in einem Abteil z.B. noch ein Rollstuhl oder Fahrrad mitgenommen werden kann. Da einer Person oder Reisegruppe ein gesamtes Abteil zugewiesen wird, kann die Mitnahme eines solchen Gegenstands garantiert werden. Die Abteile sind durch Scheiben voneinander getrennt und weden gesondert klimatisiert. Neben der höheren Masse hat der Abteilbus den Nachteil, dass nur drei Reisende bzw. Reisegruppen mitfahren können, d.h. der Kleinbus kann bis zu sechs einzelnd reisende Personen mitnehmen, der Abteilbus nur drei Personen. Der Abteilbus kann bis zu 18 Personen transportieren, soweit drei Gruppen nicht überschritten werden. Die Nachfrage, die den Simulationen zugrunde lag, hat variierende Gruppengrößen nicht berücksichtigt. Tabelle 2 gibt einen Überblick über die Parametrisierung der Fahrzeugtypen.

Die Anschaffungskosten von Fahrzeugen stellen einen wesentlichen Faktor für die Berechnung der Wirtschaftlichkeit von Geschäftsmodellen im Bereich DRT dar und sind maßgeblich für die Wettbewerbsfähigkeit im Vergleich mit konkurrierenden Mobilitätsangeboten (wie Pkw, Taxi, öffentlicher Linienverkehr). Diese werden am Beispiel eines exemplarischen Transporters der Klasse N1 (Kleinbus) und N2 (DRT-Abteilbus) über das vom DLR entwickelte Fahrzeugtechnik-Szenariomodell VECTOR21 berechnet und dargestellt. Das Modell basiert auf der Kombination eines Bottom-Up- und Discrete-Choice-Ansatzes und simuliert 
agentenbasiert individuelle Kaufentscheidungen von Flottenbetreibern vor dem Hintergrund des Wettbewerbs zwischen konventionellen und alternativen Antriebssträngen unterschiedlicher Fahrzeugsegmente für Straßentransportanwendungen. Über den Modellansatz werden angebotsseitig Fahrzeugkonzepte und -technologien sowie nachfrageseitig Flottenbetreiber unter Berücksichtigung externer Einflüsse und Verhaltensmerkmale des Verkehrsmarktes kombiniert. Zur Berechnung der Anschaffungskosten wird auf das Fahrzeug-Angebotsmodell fokussiert. Grundsätzlich beschränkt sich im Modell das Fahrzeugangebot auf die auf dem Markt derzeit und zukünftig verfügbaren Nutzfahrzeugkonzepte und Technologieoptionen. Deshalb wurden Basisfahrzeuge nach dem heutigen Stand der Technik identifiziert, die den Durchschnitt der Neuzulassungen eines ganzen Fahrzeugsegments abbilden. Die Fahrzeug-Charakteristika der betrachteten Segmente N1 (Kleinbus, 3,5t) und N2 (DRT-Abteilbus, 7,5t) wurden auf Basis einer umfassenden Literaturrecherche definiert. Sie umfassen die Definition der Fahrwiderstandsparameter wie Fahrzeugmasse, Rollwiderstands- und Luftwiderstandsbeiwert sowie die Konfiguration und Parametrisierung der Schlüsselkomponenten im jeweiligen Antriebsstrang. Um die individuellen Fahrzeugeigenschaften wie Nutzlast, Nutzvolumen, Reichweite, Energieverbrauch und Kaufpreis zu bestimmen, wird ein auf spezifische Transportanwendungen optimierendes Kostenmodell genutzt. Darüber hinaus wird für jedes Fahrzeugsegment und jede Antriebsstrangtechnologie ein Technologieportfolio bereitgestellt, das in die vier verschiedenen Kategorien Antriebsstrang, Leichtbau, Aerodynamik und weitere unterteilt ist. Es umfasst verschiedene einzelne Technologieoptionen, die die Effizienz der Fahrzeuge je nach Transportanwendung und Entwicklungspfad über die Zeit und abhängig z. B. von regulatorischer Gesetzgebung weiter verbessern.

Die beiden Transportfahrzeuge Kleinbus und DRT-Abteilbus wurden mit rein elektrischem Antriebsstrang und einer WLTP-Reichweite (Worldwide Harmonized Light Vehicles Test Procedure) von ca. 300 km ausgelegt. Die Anschaffungskosten setzen sich auf Basis von stückzahlbasierten Lernkurveneffekten in den Kategorien Karosserie/Aufbau sowie Antriebsstrang (Elektrische Maschine, Leistungselektronik, Batteriesystem) zusammen. Für alle Komponenten wird eine Kleinserie in Höhe von je 1.000 Einheiten angenommen, sodass Skaleneffekte in dieser Berechnung kaum zum Tragen kommen. Hieraus resultieren spezifische Kosten in Höhe von 28 Euro/kW für die E-Maschine, 23 Euro/kW für die Leistungselektronik und 272 Euro/kWh für das Batteriesystem. Die Produktionskosten für Karosserie/Aufbau liegen bei 18.000 Euro für den Kleinbus und 25.125 Euro für den DRT-Abteilbus. Inklusive Marge belaufen sich die Anschaffungskosten für den Kleinbus auf 94.500 Euro und für den DRT-Abteilbus auf 134.000 Euro.
Tabelle 2

Parameter von Kleinbus und Demand-Responsive Transport-Abteilbus

\begin{tabular}{lcc} 
Parameter & Kleinbus & DRT-Abteilbus \\
\hline Masse & $2.700 \mathrm{Kg}$ & $5.000 \mathrm{Kg}$ \\
\hline Länge & $5 \mathrm{~m}$ & $8 \mathrm{~m}$ \\
\hline Geschwindigkeit & $100 \mathrm{~km} / \mathrm{h}$ & $100 \mathrm{~km} / \mathrm{h}$ \\
\hline Achsen & 2 & 2 \\
\hline Antriebssystem & batterieelektrisch & batterieelektrisch \\
\hline Leistung E-Motor & $100 \mathrm{~kW}$ & $140 \mathrm{~kW}$ \\
\hline Ladeintervalle & Depot über Nacht & Depot über Nacht \\
\hline Ladeleistung & $50 \mathrm{~kW}$ & $50 \mathrm{~kW}$ \\
\hline Energiebedarf & $36 \mathrm{kWh} / 100 \mathrm{~km}$ & $51 \mathrm{kWh} / 100 \mathrm{~km}$ \\
\hline Reichweite (WLTP) & $300 \mathrm{~km}$ & $300 \mathrm{~km}$ \\
\hline Kapazität Batterie & $150 \mathrm{kWh}$ & $210 \mathrm{kWh}$ \\
\hline Automatisierung & bis SAE-Stufe 2 & bis SAE-Stufe 2 \\
\hline Abteile & 1 & 3 \\
\hline Sitzplätze je Abteil & 6 & bis zu 6 (3 klappbar) \\
\hline Sitzplätze ohne Fahrer & 6 & bis zu 18 \\
\hline Klimatisierung & Einheitliches System, & Vier getrennte Kreisläu- \\
\hline
\end{tabular}

Quelle: eigene Berechnungen.

\section{Betriebswirtschaftliche Analyse}

Ziel der Analyse ist eine Berechnung der Kosten des simulierten DRT-Betriebs eines Tages mit 100 Fahrzeugen im Vergleich der Fahrzeugtypen Kleinbus und Abteilbus. Es wurde eine Investitionsrechnung über den Zeitraum von zehn Jahren mit einem kalkulatorischen Zins von 1,7\% durchgeführt. Dabei soll der simulierte Betrieb an 365 Tagen im Jahr unverändert ablaufen und die Lebensdauer der Fahrzeuge zehn Jahre mit den zuvor ermittelten Anschaffungskosten betragen (vgl. Tabelle 3). Die jährlichen Besitzkosten der Fahrzeuge sind in Kosten für Energie, Reinigung und Instandhaltung unterteilt. Der Strompreis wurde mit 0,19 Cent je kWh angesetzt. Der mittlere Energiebedarf je Fahrzeugtyp kann Tabelle 2 entnommen werden. Dem Strombedarf für $70.000 \mathrm{~km}$ je Betriebstag werden noch $10 \%$ für Verlustleistung durch das Laden hinzugerechnet. Für den Kleinbus wurden für den Reinigungsprozess vier Minuten je Fahrzeug angesetzt, für den Abteilbus sechs Minuten. Die Instandhaltungskosten enthalten jährlich $2 \%$ der Anschaffungskosten für Wartung und Instandsetzung und zwei Reifensätze je Jahr, wobei für den schwereren Abteilbus $25 \%$ höhere Reifenkosten gegenüber dem Kleinbus angesetzt wurden.

Zur Berechnung der Personalkosten wurde ein Kostensatz von 20 Euro je Stunde angenommen. Je Fahrzeug ist für die nächtliche Ladung eine Ladesäule notwendig. Neben den Anschaffungskosten von 75.000 Euro und einer Lebensdauer von zehn Jahren wurde für die Instandhaltung jährlich 
Tabelle 3

Investitionsrechnung des Demand-Responsive Transport-Betriebs mit den unterschiedlichen Fahrzeugtypen

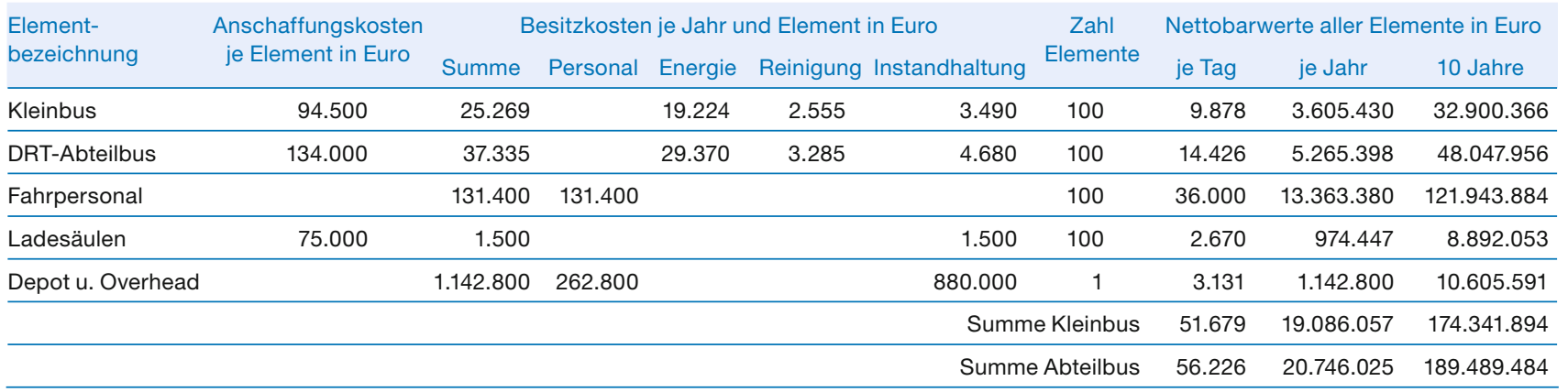

Quelle: eigene Berechnungen.

$2 \%$ der Anschaffungskosten angenommen. Die Kosten der Fahrzeugdepots und der Verwaltung des Betriebs (Overhead) wurden stark vereinfacht mit $2 \%$ der Personalkosten und 1.500 Euro je Fahrzeug und Jahr und 20 Euro je Fahrzeug und Tag angesetzt. Kosten für ein digitalisiertes Buchungs- und Dispositionssystem wurden nicht berücksichtigt, ebenso wenig wie eine differenzierte Betrachtung zur Fahrpreiserhebung. Bei einer vereinfachten Berechnung mit 1 Euro je Fahrt als Grundpreis plus 30 Cent je Fahrtkilometer wurden ca. 23.000 Euro Entgelteinnahmen je Betriebstag ermittelt. Der Abteilbus ist im direkten Fahrzeugvergleich ca. $45 \%$ kostenintensiver als der Kleinbus. Die Personalkosten sind am höchsten.

In einer Gesamtbetrachtung inklusive der weiteren Kostenpositionen liegt die Kostensteigerung des Abteilbusses gegenüber dem Kleinbus bei knapp $10 \%$, die bei einer Fahrpreisfestsetzung entsprechend berücksichtigt werden müsste. In interdisziplinären Projektteams des DLR mit Verkehrspsycholog:innen, -ingenieur:innen und -ökonom:innen wird aktuell untersucht, inwiefern potenzielle Nutzende des Abteilbusses eine Angebotsqualitätsveränderung gegenüber dem Kleinbus wahrnehmen und bereit wären, einen höheren Fahrpreis zu zahlen.

Fazit

Die Corona-Pandemie zeigt aktuell große Auswirkungen auf die Nachfrage öffentlicher Verkehrsmittel. Dass viele Menschen nicht auf engem Raum zusammenkommen möchten, ist ein Problem für den öffentlichen Verkehr, auch für die Zeit nach der Pandemie. Wie die Gesellschaft mit (subjektiv empfundenem) Infektionsrisiko umgehen wird, kann nicht vorhergesagt werden. Zumindest in ländlichen Gebieten mit geringerer Nachfrage gibt es Ansätze, diesen Nachteil in einen möglichen Vorteil zu wandeln. Den Reisenden einen exklusiv nutzbaren Raum zur Verfügung zu stellen, bietet Chancen, die Angebotsqualität des ÖPNV zu erhöhen. Gegenüber herkömmlichen Fahrzeugen sind Fahrzeuge mit zu teilendem Fahrgastraum mit Kostensteigerungen verbunden, die hier anhand eines Anwendungsbeispiels verkehrsmodellgestützt mit knapp $10 \%$ berechnet wurden. Es verbleibt die Frage, inwiefern sich die Angebotsqualität hierdurch verändern und sich dies schlussendlich auf die Nachfrage auswirken würde.

\section{Literaturverzeichnis}

Alvarez Lopez, P., M. Behrisch, L. Bieker-Walz, J. Erdmann, Y-P. Flötteröd, R. Hilbrich, L. Lücken, J. Rummel, P. Wagner und E. Wießner (2019), Microscopic Traffic Simulation using SUMO, Maui, USA: IEEE Intelligent Transportation Systems Conference (ITSC), 2572-2582.

Follmer, R. S. (2020), Mobilitätsreport 02, Ergebnisse aus Beobachtungen per repräsentativer Befragung und ergänzender Mobilitätstracking bis Ende Juni, Ausgabe 30. Juli, mit Förderung des BMBF.

Laws, R., M. Enoch, S. Ison und S. Potter (2009), Demand Responsive Transport: A Review of Schemes in England and Wales, Journal of Public Transportation, 12(1), 19-37.

Nemtanu, F., J. Schlingensiepen, D. Buretea und V. Iordache (2016), Mobility as a Service in smart cities, in A. Zbuchea und D. Nikolaidis (Hrsg.), Resonsible entrepeneurship. Vision, development and ethics: Proceedings of the 9th International conference for entrepeneurship, innovation and regional development, 23./24. Juni, 425-435.

Ronald, N., R. Thompson und S. Winter (2015), Simulating Demand-responsive Transortation: A Review of Agent-based Approaches, Transport Reviews, 35(4), 404-421.

Title: Opportunities and Potentials of Mobility as a Service After the Corona Pandemic

Abstract: The pandemic represents a break in the positive development of public transport. The users' fear of infection could have a lasting negative effect on public transport. This article presents the opportunities and potentials of mobility as a service, with a focus on demand responsive transport. Particular opportunities could lie in a vehicle concept with exclusive compartments, which also have advantages over conventional public transport vehicles in terms of infection protection. Acquisition and ownership costs are determined on the basis of an operating simulation.

JEL Classification: R42, Q55, 112 